\title{
RESPONSE OF THREE SUGAR BEET VARIETIES TO COMPOST, MINERAL NITROGEN FERTILIZER AND THEIR COMBINATION UNDER SANDY SOIL CONDITIONS I.GROWTH ATTRIBUTES
}

(Received:8.9.2011)

\author{
By \\ S. A. Safina and E. M. Abdel Fatah* \\ Agronomy Department, Faculty of Agriculture, Cairo University, Egypt and * Sugar Crops Research \\ Institute, Agriculture Research Center, Egypt
}

\begin{abstract}
Two field experiments were carried out at the Agricultural Experiments Desert Station, Faculty of Agriculture, Cairo University in Wadi El-Natroon, El-Beheira Governorate, during 2008/2009 and 2009/2010 seasons, to study the response of three sugar beet varieties, i.e. KWF1436, Swello and Faraha to compost (CM) and Mineral-N fertilizer and their combinations, at five treatments : 4 tons fed $^{-1}$ of $(\mathrm{CM}), 4$ tons fed ${ }^{-1}$ of $(\mathrm{CM})+80 \mathrm{~kg} \mathrm{~N}$ fed $^{-1}(100 \% \mathrm{~N}), 4$ tons fed $^{-1}$ of $(\mathrm{CM})+60 \mathrm{~kg} \mathrm{~N} f e d^{-1}(75 \% \mathrm{~N}), 4$ tons $\mathrm{fed}^{-1}$ of $(\mathrm{CM})+40 \mathrm{~kg} \mathrm{~N}^{-1}(50 \% \mathrm{~N})$ and $80 \mathrm{~kg} \mathrm{~N} \mathrm{fed}^{-1}(100 \% \mathrm{~N})$ on growth attributes of sugar beet under drip irrigation system. The obtained results revealed that the tested sugar beet varieties significantly differed in all the traits under study except for, top dry weight in the $1^{\text {st }}$ season and root diameter in the $2^{\text {nd }}$ one. KWS1436 variety was superior to the other two varieties in chlorophyll a and $b$ in both seasons. The highest leaf area index (LAI), root length and diameter, top and root fresh and dry weight, total dry weight were obtained by Faraha variety in both seasons. Application of $80 \mathrm{~kg} \mathrm{~N}^{-1}(100 \% \mathrm{~N})$ significantly increased the content of chlorophyll, $\mathrm{a}$ and $\mathrm{b}$ in beet leaves and gave the highest LAI and top dry weight in both seasons and the highest root length in the $1^{\text {st }}$ season. Combination of $\mathrm{CM}+80 \mathrm{~kg} \mathrm{~N}$ $\mathrm{fed}^{-1}$, recorded the highest content of carotenoids in beet leaves, root fresh and dry weight and total plant dry weight in both seasons and root diameter in the $1^{\text {st }}$ season. Various interaction orders among the two factors affected significantly all traits except for top fresh weight in the $1^{\text {st }}$ season.
\end{abstract}

Key words: compost,growth attributes, nitrogen fertilizer, sandy soil, sugar beet (Beta vulgaris, L.) varieties.

\section{INTRODUCTION}

Sugar beet (Beta vulgaris, L.) is an important cash crop for Egyptian farmers and also contributes to the local economy. Sugar beet growth is largely influenced by the agronomic practices as crop stand and fertilization, especially in the newly reclaimed soils characterized by low content of organic matter and nutrients. Many investigations have been oriented to optimize using of nitrogen through a better understanding of crop requirements under varying conditions of soil and climate. This is because nitrogen has pronounced effect on growth and physiological processes of sugar beet (Salama and Badawi, 1996; Ghura et al., 2000 and Attallah and El Etreiby 2002). Moustafa and El-Masry (2006) found that application of $120 \mathrm{~kg} \mathrm{~N} \mathrm{fed}{ }^{-1}$ significantly increased photosynthetic pigment content (chlorophyll a, b, and carotenoides) and leaf area / plant. Masri (2008) found that root fresh weight was increased with increasing $\mathrm{N}$ levels from 90 to $150 \mathrm{~kg} \mathrm{~N}$ fed $^{-1}$. Also El-Sarag (2009) reported that increasing $\mathrm{N}$ rates from 60 to120 $\mathrm{kg} \mathrm{N} \mathrm{fed}^{-1}$ increased top fresh weight by $83.3 \%$ and root fresh weight by 0.772 and 0.752 $\mathrm{kg} / \mathrm{plant}$ up to 0.853 and $0.869 \mathrm{~kg} / \mathrm{plant}$. Ferweez et al. (2011) indicated that adding $\mathrm{N}$ fertilizer at 100 or $120 \mathrm{~kg} \mathrm{~N}$ fed $^{-1}$ caused an increase in root length by 8.58 and $11.32 \%$ and root diameter by 7.78 and $11.84 \%$ compared to adding $80 \mathrm{~kg} \mathrm{~N}$ fed $^{-1}$.

Recently, some investigators tried to utilize the farmyard manure (FYM) to fertilize sugar beet to decrease the cost and minimize the pollution due to mineral fertilizers and drainage water. Furthermore, agricultural use of compost has increased due to the fact that composting represents a low-cost disposal method for organic wastes that improve the physical structure of soil. The rapid growth of organic farming has further 
accelerated the use of compost. Compost has been shown to have a positive effect on agricultural soils and crop production, because compost provides a whole array of nutrients for the soil (Seok-In and Hee-Myong, 2009). Mohamed (2008) recorded that fertilizing sugar beet by 2 ton/fed. compost produced the highest values of root length, root fresh weight and root dry weight. Also El habbasha et al. (2008) found that saline water irrigation and organic manure significantly affected most of the growth traits. Higher values of root length, diameter, fresh and dry weight and leaf fresh and dry weight were produced by 25.0 $\mathrm{m}^{3} /$ fed.

Many authors studied the difference between sugar beet varieties. Attallah (2004) evaluated ten sugar beet varieties, and recorded significant differences between them. The highest root weight was 2042.69 and $1821.68 \mathrm{~g}_{\text {plant }}{ }^{-1}$ obtained from Kawimera and Pamela, respectively. Abou El Seoud et al. (2009) tested two sugar beet varieties (Lados and TWS 1436). They found that Lados gave highly significant values compared to TWS 1436 in root length and diameter, root fresh and dry weight, top fresh and dry weight and leaf area index. In contrast, Abd El-Wahab et al. (2005) found that the studied cultivars almost did not differ significantly from each other in root length, diameter and weight.

The objectives of this research were to find out the best variety to be grown under the stress conditions (sandy soil and salinity irrigation water of 2496-2650 ppm) and the best nitrogen level with organic fertilizer to obtain the highest growth traits of sugar beet.

\section{MATERIALS AND METHODS}

Two field experiments were carried out at the Agricultural Experiments Desert Station of the Faculty of Agriculture, Cairo University in Wadi El-Natroon, El-Beheira Governorate, during the two successive winter seasons of 2008/2009 and $2009 / 2010$ to evaluate three sugar beet varieties (KWS1436, Swello and Faraha) to compost (CM) , three rates of mineral- $\mathrm{N}$ fertilizer and their combinations, at five treatments : 4 tons $\mathrm{fed}^{-1}$ of (CM), 4 tons fed ${ }^{-1}$ of $(\mathrm{CM})+80 \mathrm{~kg} \mathrm{~N}^{-1} \mathrm{fed}^{-1}(100 \%$ $\mathrm{N}), 4$ tons fed $\mathrm{f}^{-1}$ of $(\mathrm{CM})+60 \mathrm{~kg} \mathrm{~N} f e d^{-1}(75 \% \mathrm{~N})$, 4 tons fed ${ }^{-1}$ of $(\mathrm{CM})+40 \mathrm{~kg} \mathrm{~N} \mathrm{fed}^{-1}(50 \% \mathrm{~N})$ and $80 \mathrm{~kg} \mathrm{~N} \mathrm{fed}^{-1}(100 \% \mathrm{~N}$, recommended rate) on growth traits of sugar beet. Treatments were arranged in a split-plot in a randomized complete block design with three replications. The main plots were devoted to varieties, while sub plots were occupied by fertilizer treatments. Plot area was $21 \mathrm{~m}^{2}$ (6 ridges, $7 \mathrm{~cm}$ long and $50 \mathrm{~cm}$ apart). Sugar beet was sown on 10 and 15 October in the two seasons, respectively.

All plots were fertilized with $30 \mathrm{~kg} \mathrm{P}_{2} \mathrm{O}_{5} /$ fed. before planting in the form of single superphosphate $\left(15.5 \% \mathrm{P}_{2} \mathrm{O}_{5}\right)$ as one dose. $50 \mathrm{~kg} \mathrm{~K}_{2} \mathrm{O}$ $\mathrm{fed}^{-1}$ in the form of potassium sulphate $\left(48 \% \mathrm{~K}_{2} \mathrm{O}\right)$ was added through six equal doses. The first dose was added after thinning and the remaining doses were applied at 7-day intervals. Nitrogen fertilizer

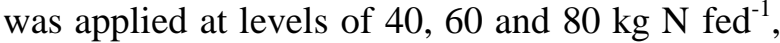
in the form of ammonium nitrate $(33.5 \% \mathrm{~N})$ in six equal doses; the first dose was added after thinning and the other doses were applied at 7day intervals. Two ton/fed. of compost (CM) was broadcasted on the soil two weeks before sowing. All suitable agricultural practices were conducted in the proper time. The mechanical and chemical analyses of the soil, water and compost analysis were carried out by the Reclamation and Development Center for desert soils, Faculty of Agriculture, Cairo University (Tables 1, 2 and 3). The two field experiments were conducted under drip irrigation system.

\subsection{Studied characters:}

After 90 days seven plants were taken randomly from each plot to determine - Leaf area index (LAI) which was calculated according to Watson (1958) and photosynthetic pigments (chlorophyll a, b and carotenoides) according to Holden (1965) after 210 days from sowing . At harvest a random sample of ten guarded plants from each plot was taken to estimate the following characters:

1-Average root dimensions [length and diameter (cm)]

2-Average root and top fresh weight ( $\mathrm{kg} / \mathrm{plant})$

3- Average root and top dry weight and total dry weight ( $\mathrm{g} /$ plant).

Data obtained from each season of the study were statistically analyzed according to the procedures outlined by Gomez and Gomez (1984) using M-STAT-C computer program (Freed et al., 1989). The differences among treatment means were compared by Least Significant Difference test (L.S.D) at 0.05 level of propability.

\section{RESULTS AND DISCUSSION 3.1. Effect of Varieties}

Data presented in Tables (4 and 5) showed that the tested sugar beet varieties responded significantly in all traits under study except for, top dry weight in the $1^{\text {st }}$ season and root diameter in the $2^{\text {nd }}$ one.

\subsubsection{Photosynthetic pigments}


Table (1): Physical and chemical properties of soil in 2008/2009 and 2009/2010 seasons.

\begin{tabular}{|c|c|c|}
\hline \multirow{2}{*}{ Soil properties } & \multicolumn{2}{|c|}{ Seasons } \\
\hline & 2008/2009 & $2009 / 2010$ \\
\hline \multicolumn{3}{|c|}{ Physical properties } \\
\hline Sand $\%$ & 93.0 & 92.25 \\
\hline Silt $\%$ & 4.56 & 5.19 \\
\hline Clay \% & 2.44 & 2.56 \\
\hline Texture & Sandy & Sandy \\
\hline \multicolumn{3}{|c|}{ Chemical properties } \\
\hline Soil (pH) & 7.81 & 7.75 \\
\hline Ec $(\mathrm{ds} / \mathrm{m})$ & 7.80 & 7.50 \\
\hline Organic Matter $(\%)$ & 0.29 & 0.32 \\
\hline Total CaCo3 (\%) & 2.59 & 2.65 \\
\hline Total N (\%) & 0.60 & 0.65 \\
\hline \multicolumn{3}{|c|}{ Soluble anions concentration (meq/L) (meq/100g soil) } \\
\hline $\mathrm{Cl}^{-}$ & 77.75 & 77.0 \\
\hline $\mathrm{HCO}_{3}^{-}$ & 0.51 & 0.55 \\
\hline $\mathrm{SO}_{4}^{-}$ & 0.52 & 0.49 \\
\hline \multicolumn{3}{|c|}{ Soluble cations concentration (meq/L) (meq/100g soil) } \\
\hline $\mathrm{Na}^{+}$ & 52.0 & 50.0 \\
\hline $\mathrm{K}^{+}$ & 1.00 & 1.20 \\
\hline $\mathrm{Ca}^{+}$ & 17.00 & 7.50 \\
\hline $\mathrm{Mg}^{+}$ & 17.00 & 18.00 \\
\hline
\end{tabular}

Table (2): Chemical analysis of water sample in 2008/2009 and 2009/2010 years.

\begin{tabular}{|c|c|c|c|c|c|c|c|c|c|c|}
\hline \multirow{2}{*}{ Year } & pH & \multicolumn{2}{|c|}{ EC } & \multicolumn{7}{|c|}{ Ions concentration meq/L } \\
\hline & Unit & $\mathrm{ds} / \mathrm{m}$ & Ppm & $\mathrm{HCO}_{3}^{-}+\mathrm{CO}_{3}^{-}$ & $\mathrm{Cl}^{-}$ & $\mathrm{SO}_{4}{ }^{=}$ & $\mathbf{C a}^{++}$ & $\mathbf{M g}^{++}$ & $\mathrm{Na}^{+}$ & $\mathbf{K a}^{+}$ \\
\hline $2008 / 2009$ & 7.49 & 3.9 & 2496 & 3.7 & 31.5 & 7.60 & 4.5 & 5.10 & 34.9 & 0.50 \\
\hline $2009 / 2010$ & 7.43 & 4.15 & 2656 & 3.2 & 30.0 & 7.10 & 5.0 & 4.0 & 30.0 & 0.42 \\
\hline
\end{tabular}

Table (3): The mean values of chemical composition and DTPA-extractable micronutrients of the used compost

\begin{tabular}{|c|c|c|c|c|c|c|c|c|c|c|c|c|}
\hline \multirow{2}{*}{$\begin{array}{c}\mathbf{E c} \\
\underset{1}{\text { Dsm }}\end{array}$} & \multirow{2}{*}{ pH } & \multirow{2}{*}{$\underset{\%}{\text { O.C }}$} & \multirow{2}{*}{$\begin{array}{c}\text { T.N. } \\
\%\end{array}$} & \multirow{2}{*}{$\begin{array}{l}P \\
\%\end{array}$} & \multirow{2}{*}{$\begin{array}{l}\mathrm{K} \\
\%\end{array}$} & \multirow{2}{*}{$\begin{array}{c}\text { C/N } \\
\text { Ratio }\end{array}$} & \multirow{2}{*}{$\begin{array}{c}\text { Ash } \\
\%\end{array}$} & \multirow{2}{*}{$\begin{array}{c}\text { OM } \\
\%\end{array}$} & $\mathbf{F e}$ & Zn & Mn & $\mathrm{Cu}$ \\
\hline & & & & & & & & & \multicolumn{4}{|c|}{$\mathrm{Mg} \mathrm{kg}^{-1}$} \\
\hline 1.90 & 7.2 & 19.1 & 1.40 & 0.30 & 0.98 & 13.64 & 80.2 & 32.65 & 45.9 & 14.3 & 36.0 & 22.4 \\
\hline
\end{tabular}

Table (4): Mean performance of three sugar beet cultivars for LAI, top fresh weight and photosynthetic pigments in $2008 / 2009$ and $2009 / 2010$ seasons.

\begin{tabular}{|c|c|c|c|c|c|}
\hline \multirow{2}{*}{ Variety } & \multirow{2}{*}{ LAI } & \multirow{2}{*}{$\begin{array}{c}\text { Top fresh } \\
\text { weight } \\
\text { (Kg/plant) }\end{array}$} & \multicolumn{3}{|c|}{ Photosynthetic pigments (mg/g f.w) } \\
\hline & & & $\begin{array}{c}\text { Chlorophyll } \\
\text { a }\end{array}$ & $\begin{array}{c}\text { Chlorophyll } \\
\text { b }\end{array}$ & Carotenoids \\
\hline \multicolumn{6}{|c|}{$2008 / 2009$} \\
\hline KWS1436 & 10.23 & 0.581 & 6.16 & 2.70 & 0.95 \\
\hline Swello & 10.31 & 0.638 & 4.64 & 2.28 & 0.94 \\
\hline Faraha & 14.11 & 0.738 & 5.11 & 2.51 & 1.36 \\
\hline $\mathbf{L S D}_{0.05}$ & 0.10 & 2.7 & 0.31 & 0.28 & 0.06 \\
\hline \multicolumn{6}{|c|}{$2009 / 2010$} \\
\hline KWS1436 & 10.05 & 0.705 & 6.10 & 2.70 & 0.94 \\
\hline Swello & 10.78 & 0.682 & 4.63 & 2.31 & 0.92 \\
\hline Faraha & 13.61 & 0.725 & 5.10 & 2.49 & 1.34 \\
\hline $\mathbf{L S D}_{0.05}$ & 0.54 & 0.6 & 0.29 & 0.27 & 0.06 \\
\hline
\end{tabular}


S. A. Safina and E. M. Abdel Fatah...................................................................

Table (5): Mean performance of three sugar beet cultivars for root length and, diameter, root fresh weight, top and root dry weight and total dry weight in 2008/2009 and 2009/2010 seasons.

\begin{tabular}{|c|c|c|c|c|c|c|}
\hline Variety & $\begin{array}{l}\text { Root length } \\
\quad(\mathrm{cm})\end{array}$ & $\begin{array}{c}\text { Root } \\
\text { diameter } \\
(\mathrm{cm})\end{array}$ & $\begin{array}{l}\text { Root fresh } \\
\text { weight } \\
\text { (kg/plant) }\end{array}$ & $\begin{array}{c}\text { Top dry } \\
\text { weight } \\
\text { (g/plant) }\end{array}$ & $\begin{array}{c}\text { Root dry } \\
\text { weight } \\
\text { (g/plant) }\end{array}$ & $\begin{array}{c}\text { Total dry } \\
\text { weight } \\
\text { (g/plant) }\end{array}$ \\
\hline \multicolumn{7}{|c|}{$2008 / 2009$} \\
\hline KWS1436 & 23 & 11.80 & 1.173 & 71.50 & 240.1 & 311.60 \\
\hline Swello & 23 & 11.80 & 1.080 & 72.10 & 234.2 & 306.30 \\
\hline Faraha & 25 & 13.20 & 1.473 & 94.80 & 305.2 & 400.00 \\
\hline LSD $_{0.05}$ & 1.0 & 0.10 & 0.01 & N.S. & 1.3 & 1.30 \\
\hline \multicolumn{7}{|c|}{$2009 / 2010$} \\
\hline KWS1436 & 24 & 12.30 & 1.300 & 84.10 & 275.40 & 359.50 \\
\hline Swello & 21 & 12.30 & 1.267 & 74.80 & 253.10 & 327.90 \\
\hline Faraha & 25 & 12.50 & 1.384 & 85.70 & 282.80 & 368.50 \\
\hline $\mathbf{L S D}_{0.05}$ & 1.0 & N.S. & 0.01 & 0.80 & 0.80 & 1.40 \\
\hline
\end{tabular}

N.S.=not significant

Table (6): Effect of fertilizer treatments on LAI, top fresh weight and photosynthetic pigments in 2008/2009 and 2009/2010 seasons.

\begin{tabular}{|c|c|c|c|c|c|}
\hline \multirow[b]{2}{*}{ Fertilizer } & \multirow[b]{2}{*}{ LAI } & \multirow{2}{*}{$\begin{array}{c}\text { Top fresh } \\
\text { weight } \\
\text { (kg/plant) }\end{array}$} & \multicolumn{3}{|c|}{ Photosynthetic pigments (mg/g f.w) } \\
\hline & & & Chlorophyll a & $\begin{array}{c}\text { Chlorophyll } \\
\text { b }\end{array}$ & Carotenoides \\
\hline \multicolumn{6}{|c|}{$2008 / 2009$} \\
\hline Compost $\mathrm{CM}$ ) & 11.71 & 0.572 & 5.24 & 2.40 & 0.98 \\
\hline $\mathrm{CM}+80 \mathrm{~kg} \mathrm{~N}$ & 12.04 & 0.714 & 4.90 & 2.26 & 1.37 \\
\hline $\mathrm{CM}+60 \mathrm{~kg} \mathrm{~N}$ & 10.55 & 0.653 & 4.89 & 2.33 & 1.05 \\
\hline $\mathrm{CM}+40 \mathrm{~kg} \mathrm{~N}$ & 10.64 & 0.625 & 5.49 & 2.66 & 1.36 \\
\hline $80 \mathrm{~kg} \mathrm{~N}$ & 12.81 & 0.700 & 6.00 & 2.82 & 0.66 \\
\hline $\mathrm{LSD}_{0.05}$ & 0.07 & N.S. & 0.25 & 0.13 & 0.05 \\
\hline \multicolumn{6}{|c|}{$2009 / 2010$} \\
\hline Compost CM) & 9.29 & 0.471 & 5.22 & 2.42 & 0.97 \\
\hline $\mathrm{CM}+80 \mathrm{~kg} \mathrm{~N}$ & 11.55 & 0.795 & 4.89 & 2.28 & 1.35 \\
\hline $\mathrm{CM}+60 \mathrm{~kg} \mathrm{~N}$ & 10.47 & 0.756 & 4.88 & 2.35 & 1.04 \\
\hline $\mathrm{CM}+40 \mathrm{~kg} \mathrm{~N}$ & 12.54 & 0.756 & 5.41 & 2.66 & 1.33 \\
\hline $80 \mathrm{~kg} \mathrm{~N}$ & 13.56 & 0.742 & 5.99 & 2.79 & 0.64 \\
\hline $\operatorname{LSD}_{0.05}$ & 0.19 & 0.2 & 0.23 & 0.11 & 0.04 \\
\hline
\end{tabular}

Table (7): Effect of fertilizer treatments on root length and diameter, root fresh weight, top and root dry weight and total dry weight in 2008/2009 and 2009/2010 seasons.

\begin{tabular}{|c|c|c|c|c|c|c|}
\hline Fertilizer & $\begin{array}{l}\text { Root } \\
\text { length } \\
\text { (cm) }\end{array}$ & $\begin{array}{c}\text { Root } \\
\text { diameter } \\
(\mathrm{cm})\end{array}$ & $\begin{array}{l}\text { Root fresh } \\
\text { weight } \\
\text { (kg/plant) }\end{array}$ & $\begin{array}{l}\text { Top dry } \\
\text { weight } \\
\text { (g/plant) }\end{array}$ & $\begin{array}{c}\text { Root dry } \\
\text { weight } \\
\text { (g/plant) }\end{array}$ & $\begin{array}{c}\text { Total dry } \\
\text { weight } \\
\text { (g/plant) }\end{array}$ \\
\hline \multicolumn{7}{|c|}{$2008 / 2009$} \\
\hline Compost CM) & 22 & 11.5 & 1.11 & 69.5 & 239.9 & 309.4 \\
\hline $\mathrm{CM}+80 \mathrm{~kg} \mathrm{~N}$ & 24 & 13.2 & 1.80 & 84.8 & 306.9 & 391.6 \\
\hline $\mathrm{CM}+60 \mathrm{~kg} \mathrm{~N}$ & 23 & 12.5 & 1.11 & 81.5 & 269.4 & 350.9 \\
\hline $\mathrm{CM}+40 \mathrm{~kg} \mathrm{~N}$ & 22 & 11.5 & 1.02 & 74.3 & 242.0 & 328.3 \\
\hline $80 \mathrm{~kg} \mathrm{~N}$ & 26 & 12.5 & 1.17 & 87.4 & 240.8 & 316.1 \\
\hline LSD $_{0.05}$ & 0.1 & 0.1 & 0.01 & 0.1 & 0.1 & 0.1 \\
\hline \multicolumn{7}{|c|}{$2009 / 2010$} \\
\hline Compost CM) & 21 & 9.1 & 0.88 & 54.7 & 198.7 & 253.4 \\
\hline $\mathrm{CM}+80$ kg N & 25 & 13.4 & 1.49 & 81.2 & 346.9 & 428.0 \\
\hline $\mathrm{CM}+60 \mathrm{~kg} \mathrm{~N}$ & 24 & 12.0 & 1.46 & 86.3 & 271.1 & 359.4 \\
\hline $\mathrm{CM}+40 \mathrm{~kg} \mathrm{~N}$ & 25 & 13.1 & 1.32 & 91.4 & 269.6 & 361.0 \\
\hline $80 \mathrm{~kg} \mathrm{~N}$ & 23 & 14.2 & 1.44 & 94.1 & 265.9 & 357.9 \\
\hline LSD $_{0.05}$ & 0.7 & 0.4 & 0.01 & 0.1 & 0.1 & 0.1 \\
\hline
\end{tabular}


Table (8): Effect of interaction between sugar beet varieties and fertilizer treatmants on chlorophyll a, chlorophyll b and carotenoides (mg/g f.w.) in 2008/2009 and 2009/2010 seasons.

\begin{tabular}{|c|c|c|c|c|c|c|c|}
\hline \multirow{2}{*}{ Variety } & \multirow{2}{*}{$\begin{array}{l}\text { Fertilizer } \\
\text { treatment }\end{array}$} & \multicolumn{2}{|c|}{$\begin{array}{c}\text { Chlorophyll a } \\
\text { (mg/g f.w.) }\end{array}$} & \multicolumn{2}{|c|}{$\begin{array}{c}\text { Chlorophyll b } \\
\text { (mg/g f.w.) }\end{array}$} & \multicolumn{2}{|c|}{$\begin{array}{c}\text { Carotenoides } \\
\text { (mg/g f.w.) }\end{array}$} \\
\hline & & 2009 & 2010 & 2009 & 2010 & 2009 & 2010 \\
\hline \multirow{5}{*}{ KWS-1436 } & Compost (CM) & 5.78 & 5.81 & 2.47 & 2.54 & 0.81 & 0.78 \\
\hline & $\mathrm{CM}+80 \mathrm{~kg} \mathrm{~N}$ & 5.33 & 5.34 & 2.18 & 2.17 & 1.01 & 1.01 \\
\hline & CM+60 kg N & 6.82 & 6.77 & 3.01 & 3.02 & 0.75 & 0.77 \\
\hline & $\mathrm{CM}+40 \mathrm{~kg} \mathrm{~N}$ & 7.63 & 7.56 & 3.19 & 3.16 & 1.30 & 1.30 \\
\hline & $80 \mathrm{~kg} N$ & 5.24 & 5.03 & 2.66 & 2.62 & 0.89 & 0.87 \\
\hline \multirow{5}{*}{ Swello } & Compost (CM) & 4.85 & 4.79 & 2.24 & 2.23 & 1.42 & 1.43 \\
\hline & $\mathrm{CM}+80 \mathrm{~kg} \mathrm{~N}$ & 4.22 & 4.19 & 2.06 & 2.11 & 1.45 & 1.44 \\
\hline & CM+60 kg N & 3.48 & 3.53 & 1.84 & 1.88 & 0.87 & 0.84 \\
\hline & CM+40 kg N & 4.56 & 4.58 & 2.27 & 2.31 & 0.70 & 0.66 \\
\hline & $80 \mathrm{~kg} \mathrm{~N}$ & 6.09 & 6.08 & 2.97 & 3.01 & 0.27 & 0.24 \\
\hline \multirow{5}{*}{ Faraha } & Compost (CM) & 5.09 & 5.06 & 2.50 & 2.50 & 0.71 & 0.72 \\
\hline & CM+80 kg N & 5.15 & 5.14 & 2.55 & 2.58 & 1.63 & 1.60 \\
\hline & CM+60 kg N & 4.37 & 4.34 & 2.13 & 2.15 & 1.54 & 1.52 \\
\hline & $\mathrm{CM}+40 \mathrm{~kg} \mathrm{~N}$ & 5.82 & 5.83 & 2.53 & 2.52 & 2.08 & 2.04 \\
\hline & $80 \mathrm{~kg} \mathrm{~N}$ & 5.14 & 5.13 & 2.83 & 2.73 & 0.83 & 0.82 \\
\hline \multicolumn{2}{|c|}{$\mathbf{L S D}_{0.05}$} & 0.43 & 0.39 & 0.23 & 0.19 & 0.09 & 0.08 \\
\hline
\end{tabular}

Table (9): Effect of interaction between sugar beet varieties and fertilizer treatmants on LAI and top fresh weight in 2008/2009 and 2009/2010 seasons.

\begin{tabular}{|c|c|c|c|c|c|}
\hline \multirow{2}{*}{ Variety } & \multirow{2}{*}{$\begin{array}{l}\text { Fertilizer } \\
\text { treatment }\end{array}$} & \multicolumn{2}{|c|}{ LAI } & \multicolumn{2}{|c|}{$\begin{array}{l}\text { Top fresh weight } \\
\text { (g/plant) }\end{array}$} \\
\hline & & 2009 & 2010 & 2009 & 2010 \\
\hline \multirow{5}{*}{ KWS-1436 } & Compost (CM) & 8.35 & 6.66 & 623.7 & 475.2 \\
\hline & $\mathrm{CM}+80 \mathrm{~kg} \mathrm{~N}$ & 18.25 & 15.87 & 783.7 & 966.3 \\
\hline & $\mathrm{CM}+60 \mathrm{~kg} \mathrm{~N}$ & 13.7 & 12.21 & 642.7 & 912.2 \\
\hline & $\mathrm{CM}+40 \mathrm{~kg} \mathrm{~N}$ & 5.28 & 8.32 & 448.7 & 700.2 \\
\hline & $80 \operatorname{kg} N$ & 5.54 & 7.20 & 407.7 & 469.4 \\
\hline \multirow{5}{*}{ Swello } & Compost (CM) & 9.22 & 6.59 & 480.0 & 400.1 \\
\hline & $\mathrm{CM}+80 \mathrm{~kg} \mathrm{~N}$ & 8.81 & 11.29 & 657.0 & 739.0 \\
\hline & $\mathrm{CM}+60 \mathrm{~kg} \mathrm{~N}$ & 6.17 & 4.89 & 522.0 & 490.0 \\
\hline & $\mathrm{CM}+40 \mathrm{~kg} \mathrm{~N}$ & 15.79 & 17.52 & 768.0 & 850.1 \\
\hline & $80 \mathrm{~kg} \mathrm{~N}$ & 11.57 & 13.60 & 764.0 & 933.0 \\
\hline \multirow{5}{*}{ Faraha } & Compost (CM) & 17.56 & 14.61 & 612.0 & 537.2 \\
\hline & $\mathrm{CM}+80 \mathrm{~kg} \mathrm{~N}$ & 9.05 & 7.49 & 700.0 & 678.1 \\
\hline & $\mathrm{CM}+60 \mathrm{~kg} \mathrm{~N}$ & 11.77 & 14.30 & 793.0 & 866.2 \\
\hline & $\mathrm{CM}+40 \mathrm{~kg} \mathrm{~N}$ & 10.85 & 11.79 & 657.0 & 718.3 \\
\hline & $80 \mathrm{~kg} \mathrm{~N}$ & 21.31 & 19.87 & 623.7 & 823.2 \\
\hline \multicolumn{2}{|c|}{$\mathbf{L S D}_{0.05}$} & 0.12 & 0.34 & N.S. & 0.3 \\
\hline
\end{tabular}

N.S.= not significant 
Table (10): Effect of interaction between sugar beet varieties and fertilizer treatmants on root fresh weight and root length and diameter in 2008/2009 and 2009/2010 seasons.

\begin{tabular}{|c|c|c|c|c|c|c|c|}
\hline \multirow{2}{*}{ Variety } & \multirow{2}{*}{$\begin{array}{l}\text { Fertilizer } \\
\text { treatment }\end{array}$} & \multicolumn{2}{|c|}{$\begin{array}{c}\text { Root fresh weight } \\
(\mathrm{kg})\end{array}$} & \multicolumn{2}{|c|}{ Root length (cm) } & \multicolumn{2}{|c|}{ Root diameter $(\mathbf{c m})$} \\
\hline & & 2009 & 2010 & 2009 & 2010 & 2009 & 2010 \\
\hline \multirow{5}{*}{ KWS-1436 } & Compost $(\mathrm{CM})$ & 1.000 & 0.705 & 22 & 20 & 10.2 & 9.0 \\
\hline & $\mathrm{CM}+80 \mathrm{~kg} \mathrm{~N}$ & 2.282 & 2.031 & 29 & 30 & 15.2 & 16.0 \\
\hline & $\mathrm{CM}+60 \mathrm{~kg} \mathrm{~N}$ & 1.076 & 1.808 & 20 & 24 & 13.2 & 13.2 \\
\hline & $\mathrm{CM}+40 \mathrm{~kg} \mathrm{~N}$ & 0.704 & 1.033 & 19 & 23 & 9.2 & 11.2 \\
\hline & $80 \mathrm{~kg} N$ & 0.805 & 0.922 & 21 & 23 & 11.2 & 12.1 \\
\hline \multirow{5}{*}{ Swello } & Compost (CM) & 1.078 & 0.831 & 21 & 18 & 11.2 & 8.2 \\
\hline & $\mathrm{CM}+80 \mathrm{~kg} \mathrm{~N}$ & 1.128 & 1.426 & 20 & 23 & 11.2 & 13.2 \\
\hline & $\mathrm{CM}+60 \mathrm{~kg} \mathrm{~N}$ & 0.645 & 0.613 & 21 & 18 & 10.2 & 8.8 \\
\hline & $\mathrm{CM}+40 \mathrm{~kg} \mathrm{~N}$ & 1.304 & 1.454 & 21 & 23 & 13.2 & 14.1 \\
\hline & $80 \mathrm{~kg} \mathbf{N}$ & 1.247 & 2.011 & 28 & 24 & 13.2 & 17.3 \\
\hline \multirow{5}{*}{ Faraha } & Compost (CM) & 1.244 & 1.091 & 22 & 24 & 13.2 & 10.1 \\
\hline & $\mathrm{CM}+80 \mathrm{~kg} \mathrm{~N}$ & 2.003 & 1.013 & 23 & 21 & 13.2 & 11.0 \\
\hline & CM+60 kg N & 1.602 & 1.967 & 27 & 29 & 14.2 & 14.0 \\
\hline & $\mathrm{CM}+40 \mathrm{~kg} \mathrm{~N}$ & 1.051 & 1.473 & 24 & 28 & 12.2 & 14.0 \\
\hline & 80 kg N & 1.463 & 1.374 & 27 & 24 & 13.2 & 13.2 \\
\hline \multicolumn{2}{|c|}{ LSD $_{0.05}$} & 0.017 & 0.016 & 1.0 & 1.0 & 0.1 & 0.6 \\
\hline
\end{tabular}

Table (11): Effect of interaction between sugar beet varieties and fertilizer treatmants on top and root dry weight and total dry weight in $2008 / 2009$ and $2009 / 2010$ seasons.

\begin{tabular}{|c|c|c|c|c|c|c|c|}
\hline \multirow{2}{*}{ Variety } & \multirow{2}{*}{$\begin{array}{l}\text { Fertilizer } \\
\text { treatment }\end{array}$} & \multicolumn{2}{|c|}{$\begin{array}{c}\text { Top dry weight } \\
\text { (g/plant) }\end{array}$} & \multicolumn{2}{|c|}{$\begin{array}{c}\text { Root dry weight } \\
\text { (g/plant) }\end{array}$} & \multicolumn{2}{|c|}{$\begin{array}{c}\text { Total dry weight } \\
\text { (g/plant) }\end{array}$} \\
\hline & & 2009 & 2010 & 2009 & 2010 & 2009 & 2010 \\
\hline \multirow{5}{*}{ KWS-1436 } & Compost (CM) & 63.9 & 62.4 & 200.5 & 197.9 & 264.3 & 260.3 \\
\hline & CM+80 kg N & 94.0 & 104.3 & 322.4 & 457.0 & 416.3 & 561.3 \\
\hline & $\mathrm{CM}+60 \mathrm{~kg} \mathrm{~N}$ & 85.7 & 100.4 & 316.6 & 329.9 & 402.3 & 430.3 \\
\hline & $\mathrm{CM}+40 \mathrm{~kg} \mathrm{~N}$ & 62.7 & 84.0 & 176.1 & 201.5 & 238.8 & 285.5 \\
\hline & $80 \mathrm{~kg} \mathrm{~N}$ & 51.4 & 69.6 & 185.0 & 190.8 & 236.4 & 260.3 \\
\hline \multirow{5}{*}{ Swello } & Compost (CM) & 63.4 & 40.2 & 239.7 & 196.1 & 303.1 & 236.3 \\
\hline & $\mathrm{CM}+80 \mathrm{~kg} \mathrm{~N}$ & 67.7 & 80.6 & 243.8 & 308.0 & 311.4 & 388.6 \\
\hline & CM+60 kg N & 60.1 & 55.7 & 145.6 & 137.3 & 205.7 & 193.0 \\
\hline & $\mathrm{CM}+40 \mathrm{~kg} \mathrm{~N}$ & 86.8 & 98.3 & 292.2 & 302.4 & 379.0 & 400.7 \\
\hline & $80 \mathrm{~kg} N$ & 82.6 & 99.1 & 249.5 & 321.8 & 332.1 & 420.9 \\
\hline \multirow{5}{*}{ Faraha } & Compost (CM) & 81.1 & 61.6 & 279.6 & 202.2 & 360.7 & 263.7 \\
\hline & $\mathrm{CM}+80 \mathrm{~kg} \mathrm{~N}$ & 92.7 & 58.6 & 354.4 & 275.6 & 447.1 & 334.2 \\
\hline & CM+60 kg N & 98.8 & 102.7 & 346.0 & 346.2 & 444.8 & 448.9 \\
\hline & $\mathrm{CM}+40 \mathrm{~kg} \mathrm{~N}$ & 73.4 & 92.0 & 257.8 & 304.9 & 331.2 & 396.9 \\
\hline & 80 kg N & 128.1 & 113.6 & 287.8 & 285.1 & 415.9 & 398.7 \\
\hline \multicolumn{2}{|c|}{$\mathbf{L S D}_{0.05}$} & 0.1 & 0.2 & 0.1 & 0.1 & 0.2 & 0.1 \\
\hline
\end{tabular}


KWS1436 variety was superior compared to the other two varieties for chlorophyll, a and $b$ in the two seasons. While, Faraha variety surpassed the KWS1436 and Swello varieties in carotenoids in both seasons (Table 4).

Growth characters

Data in Tables (4 and 5) cleared that the highest leaf area index (LAI), root length and diameter, top and root fresh and dry weight and total dry weight were obtained by Faraha variety in both seasons. Differences among sugar beet varieties for LAI, top and root fresh and dry weight and total dry weight were also detected by Mohamed (2008). Ouda (2009) showed that root length and diameter of the variety Lados were significantly higher than Athose poly. Also, AlLabbody (2003) found differences among sugar beet varieties in root length and diameter. It is important to report that the differences between KWS1436 and Swello varieties were insignificant in LAI and root length and diameter in the $1^{s t}$ season.

\subsection{Effect of fertilizer treatments \\ 3.2.1. Photosynthetic pigments}

Data presented in Table (6) indicated that application of $80 \mathrm{~kg} \mathrm{~N}^{\mathrm{fed}}{ }^{-1}(100 \% \mathrm{~N})$ was more effective and significantly increased the contents of chlorophyll, $\mathrm{a}$ and $\mathrm{b}$ in beet leaves in comparison to the other treatments in both seasons. Also, it was noticed that, all combined treatments significantly increased carotenoid content as compared with using compost or Mineral-N fertilizer alone in the two seasons in favor of the combination of $\mathrm{CM}+80 \mathrm{~kg} \mathrm{~N}^{\mathrm{N}} \mathrm{fe}^{-1}$ which produced the highest content of carotenoids in the two seasons. These results may be due to the role of nitrogen in increasing the vegetative growth of sugar beet plants. These results are in agreement with Moustafa and El-Masry (2006) who reported that $\mathrm{N}$ fertilizer increased significantly photosynthetic pigments (chlorophyll, a, b and carotenoids).

\subsubsection{Growth characters}

Results in Tables (6 and 7) cleared that, all traits under study were significantly affected by $\mathrm{N}$ treatment in both seasons except top fresh weight in the $1^{\text {st }}$ season. Application of $80 \mathrm{~kg} \mathrm{~N}$ fed $^{-1}$ recorded the maximum LAI and top dry weight in both seasons and the highest root length in the $1^{\text {st }}$ season.

Application of $\mathrm{CM}+80 \mathrm{~kg} \mathrm{~N}^{-1} \mathrm{fed}^{-1}$ give the highest root fresh and dry weight and total dry weight in both seasons and significantly increased

root fresh weight by $53.85 \%$ and $3.47 \%$, root dry weight $27.45 \%$ and $30.46 \%$ and total dry weight by $23.88 \%$ and $19.59 \%$ over adding $80 \mathrm{~kg}$ $\mathrm{N} \mathrm{fed}{ }^{-1}$ in the $1^{s t}$ and $2^{\text {nd }}$ seasons, respectively.

This result may be due to applying organic manure (compost) to sandy soil which plays an important role for improving soil media throughout modifying the pore size distribution and consequently the majority of soil physical properties which is reflected in higher crop production (Badwy, 2008). Application of compost with $\mathrm{N}$ increased root length in the $2^{\text {nd }}$ season and root dry weight and total dry weight in both seasons as compared with using compost or Mineral-N alone. In combined treatments increasing $\mathrm{N}$ levels from $40 \mathrm{~kg} \mathrm{~N}^{-1} \mathrm{f}^{-1}(50 \% \mathrm{~N})$ to $80 \mathrm{~kg} \mathrm{~N} \mathrm{fed}^{-1}(100 \% \mathrm{~N})$ significantly increased the values of root length, fresh and dry weight and total dry weight in both seasons.

\subsection{Interaction effects}

Varieties and fertilizer treatment interactions affect significantly all the studied characters in both seasons except top fresh weight in the $1^{\text {st }}$ season (Tables 8, 9, 10 and 11).

\subsubsection{Photosynthetic pigments}

Using CM +40 kg N with KWS1436 variety gave the highest values of chlorophyll, $\mathrm{a}$ and $\mathrm{b}$ (7.63, 7.56 and $3.19,3.16 \mathrm{mg} / \mathrm{g}$ f.w.). While the highest values of carotenoids $(2.08$ and $2.04 \mathrm{mg} / \mathrm{g}$ f.w.) were obtained by applying $\mathrm{CM}+40 \mathrm{Kg} \mathrm{N}$ to Faraha variety, respectively in the $1^{\text {st }}$ and $2^{\text {nd }}$ seasons (Table 8).

\subsubsection{Growth characters}

The Results in Tables ( 9 and 11 ) showed that, applying $\mathrm{N}$ fertilizer at the rate of $80 \mathrm{~kg} \mathrm{~N}$ fed $^{-1}$ to the variety Faraha gave the highest LAI (21.31 and 19.87) and top dry weight (128.10 and 113.60 $\mathrm{g} / \mathrm{plant})$ in the $1^{\text {st }}$ and $2^{\text {nd }}$ season, respectively . While, KWS1436 variety which received CM + $80 \mathrm{~kg} \mathrm{~N} \mathrm{fed}{ }^{-1}$ recorded the highest, root fresh weight (2.28 and $2.03 \mathrm{~kg}$ ) and root length (29 and $30 \mathrm{~cm}$ ) in the $1^{s t}$ and $2^{\text {nd }}$ seasons, respectively (Table 10) and root dry weight and total dry weight amounted to (457.00 and $561.30 \mathrm{~g} / \mathrm{plant})$, respectively in the $2^{\text {nd }}$ season (Tables 11 ).

\section{REFERENCES}

Abd El-Wahab A.M., Abdel-Mottaleb H.M. and Yussef M.S.H. (2005). Response of some sugar beet cultivars to two fertilization treatments under sprinkler and drip irrigation systems in newly reclaimed lands, Sina; the $11^{\text {th }}$ Conf. of Agron., Agron. Dept., Fac. Agric., Assiut Univ., 15-16 Nov. 2005.

Abou El-Seoud I.I.A., Badr E.A. and Elshimaa A.E. (2009). Response of two sugar beet 
varieties to Chicken Manure and phosphorine application. Alex. Sci. Exch. J., 30 (4): 433 444.

Attallah M.Z. (2004). Effect of biofertilizer, city garbage compost, mineral-N and their combinations on productivity of sugar beet cultivars. J. Agric. Sci. Mansoura Univ., 29(7): 4281-4294.

Attallah M.Z. and El Etreiby F. (2002). The effect of compost and mineral-N on soil properties, ten sugar beet varieties and nutrient contents. Alex. Sci. Exch., 23 (1): 109-120.

Al-Labbody A.H.S. (2003). Evaluation of some multigerm and monogerm sugar beet varieties under Fayoum Governorate conditions. Ph.D. Thesis, Agron. Dept., Fac. Agric., Al-Azhar Univ.

Badwy A.A. (2008). Effect of water stress and some conditioners on the productivity of peanut crop and water relation in sandy soil. J. Biol. Chem. Environ. Sci., 3 (1): 445-454.

El- Habbasha S. F., Okasha E. M. and Abdou M. A. A. (2008). Response of sugar beet (Beta vulgaris, $L$.) to irrigation with saline water, organic manure and soil texture. Egypt . J. Agron. 30 (2), pp. 201-215.

El-Sarag E.I. (2009). Maximizing sugar beet yield, quality and water use efficiency using some agricultural practices in North Sinai conditions. Bull. Fac. Agric. Cairo Univ. 60: 155-167.

Ferweez H. Ibrahim M. F. M. and Allan A. M. (2011). Improving yield and quality of sugar beet using boron at different levels of nitrogen fertilizer. Alex. Sci., Exch., 32(1) 51-57.

Freed R., Einensmith S.P., Gutez S., Reicosky D., Smail V.W. and Wolberg P. (1989). Guide to MSTAT-C Analysis of Agronomic Research Experiments. Michigan Univ., East Lansing, U.S.A.

Ghura N.S., Attallah M.Z. and Amer M. (2000). Effect of NPK treatments on yield differential gene action and chemical compostion of three sugar beet varieties. Alex. Sci. Exch., 21(4): 293-310.

Gomez K.A. and Gomez A.A. (1984). Statistical Procedures for Agricultural Research $2^{\text {nd }}$ ed. (Eds. John Willey and Sons), New York, USA. 215-225.

Holden M.(1965).In Chemistry and biochemistry of plant pigment. Ed., Goodwin, T.W., pp 462-88. (Academic Press, London).

Masri M. I. (2008). Effect of nitrogen level and planting density on sugar beet yield and its attributes. Egypt . J. Agron. 30 (2), pp. 119136.

Mohamed H.Y. (2008). Influence of some biochemical fertilization regimes and organic fertilizer on yield and quality of some sugar beet varieties. Ph.D. Thesis, Fac. Agric., Zagazig Univ., Egypt.

Moustafa Sh.N. and El-Masry A.A. (2006). Effect of nitrogen and potassium fertilization with or without spraying by iron combined with manganese on some physio-chemical properties, productivity and quality of sugar beet crop. Annals of Agric. Sci., Moshtohor, 44(4): 1431-1446.

Ouda S.M.M. (2009). Yield and quality of two sugar beet varieties as influenced by nitrogen fertigation regimes under dripirrigation system. J. Agric. Sci. Mansoura Univ., 34 (4): 3189-3198.

Seok-In Yun and Hee-Myong Ro (2009). Natural $15 \mathrm{~N}$ abundance of plant and soil inorganic-N as evidence for over-fertilization with compost. Soil Biology \& Biochemistry, 41: 1541-1547.

Salama A.A. and Badawi M.A (1996). Evaluation of six sugar beet cultivars under nitrogen levels and harvesting dates. J. Agric. Sci. Mansoura Univ., 21(1): 139-153.

Watson D.J. (1958). The dependence of net assimilation rate on leaf area index. Ann. Bot. N.S. 22: 37-54. 


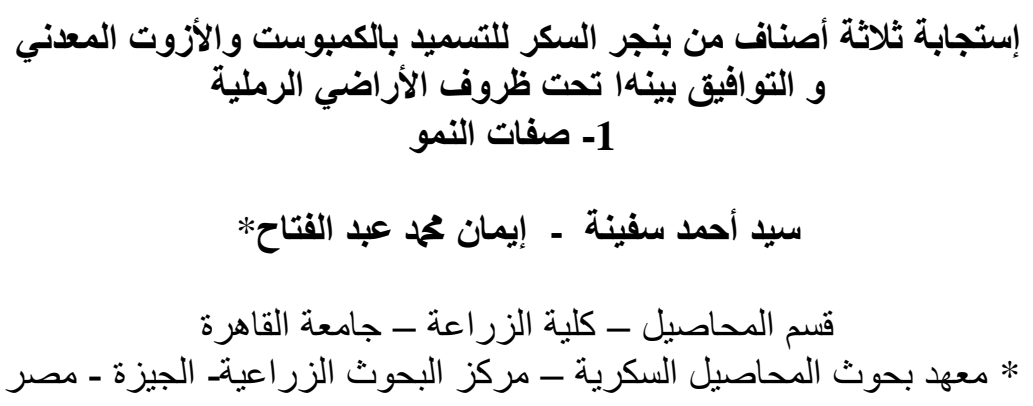

أجريت تجربتان حقليتان بمحطة التجارب الزر اعية الصحر اوية لكلبة الزر اعة جامعة القاهرة بوادي النطرون،

بمحافظة البحيرة ، خلال موسمي 2009/2008 ، (Swello and Faraha

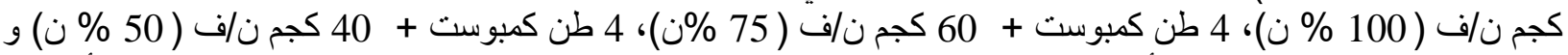

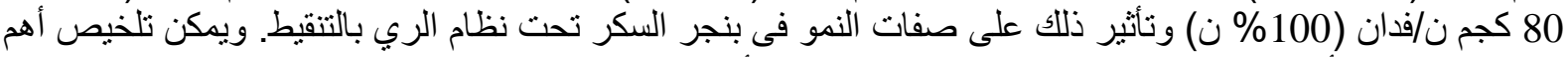

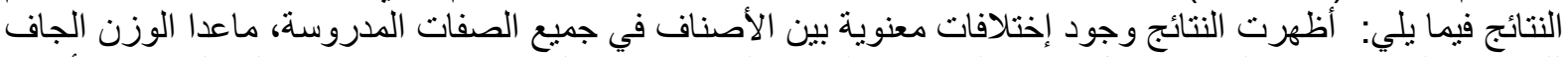

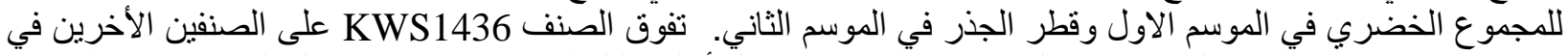

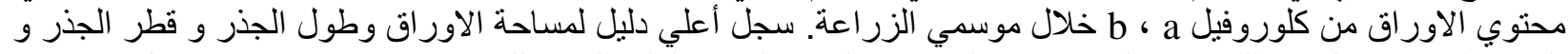

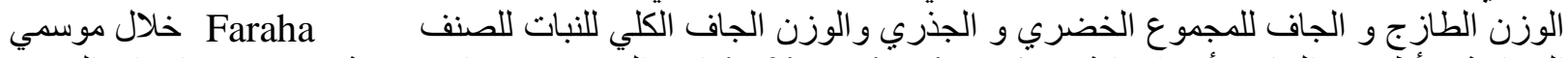

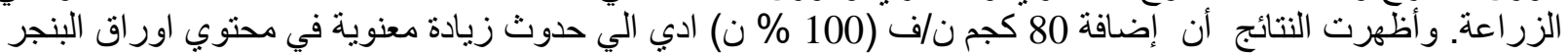

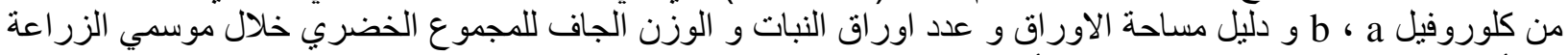

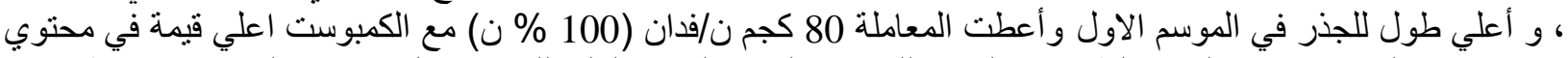

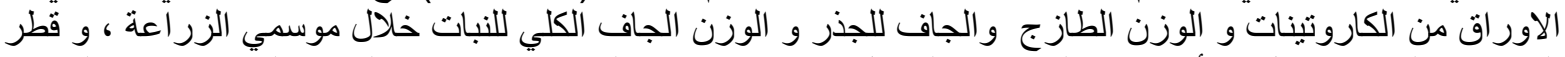

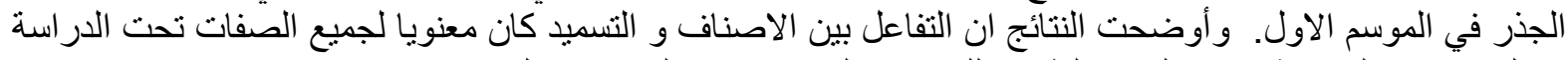

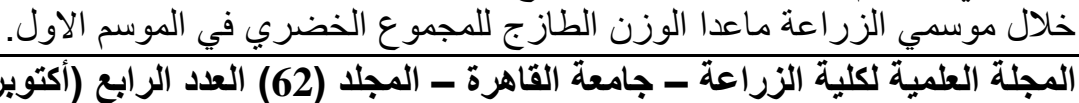

